\title{
Effectiveness of Remdesivir, Lopinavir/Ritonavir, and Favipiravir for COVID-19 Treatment: A Systematic Review
}

\author{
Windi Fresha Qomara (D) \\ Delya Nur Primanissa (D) \\ Salma Hasni Amalia I ${ }^{\prime}$ \\ Febby $V$ Purwadi (iD) \\ Neily Zakiyah (D) ${ }^{1,2}$ \\ 'Center of Excellence in Higher \\ Education for Pharmaceutical Care \\ Innovation, Universitas Padjadjaran, \\ Bandung, 40I32, Indonesia; ${ }^{2}$ Department \\ of Pharmacology and Clinical Pharmacy, \\ Faculty of Pharmacy, Universitas \\ Padjadjaran, Bandung, 40I32, Indonesia
}

Correspondence: Neily Zakiyah Department of Pharmacology and Clinical Pharmacy, Faculty of Pharmacy, Universitas Padjadjaran, Bandung, 40I32, Indonesia

Tel +62-22-77962

Email neily.zakiyah@unpad.ac.id
Background: The severe acute respiratory syndrome coronavirus 2 (SARS-CoV-2) is a novel strain that causes acute respiratory illnesses known as coronavirus disease 2019 (COVID-19). Currently, there is limited information regarding the therapeutic management for this disease. Several studies have stated that antivirals drugs such as remdesivir, favipiravir, and lopinavir/ritonavir may potentially inhibit the virus from spreading to the host.

Objective: The aim of this systematic review was to summarize the clinical effectiveness and safety of remdesivir, favipiravir, and lopinavir/ritonavir on COVID-19.

Methods: The PubMed and Cochrane Library databases were searched up to July 2021 to identify eligible experimental randomized controlled trials on remdesivir, favipiravir, and lopinavir/ritonavir for COVID-19 patients. This systematic review followed the Preferred Reporting Items for Systematic Reviews and Meta-Analyses (PRISMA) guideline.

Results: From 158 references, 15 studies were included in the review. The results showed that remdesivir has some potential benefits for hospitalized COVID-19 patients, as seen from clinical improvements such as faster recovery time, less duration of hospitalization, and fewer respiratory side effects among COVID-19 patients. However, the impact of remdesivir in reducing mortality remains uncertain. Treatment with favipiravir has shown promising improvement in the clinical status of COVID-19 patients, although the results suggested no significant differences in some clinical parameters such as length of hospitalizations and clinical recovery. A combination of favipiravir with other supportive therapy showed more favorable outcomes for COVID-19 patients. Furthermore, the use of lopinavir/ritonavir in COVID-19 patients reported no significant clinical improvement compared to standard care with notable adverse effect reactions.

Conclusion: This study provides an overview of the evidence-based role of remdesivir, favipiravir, and lopinavir/ritonavir in the management of COVID-19. A thorough assessment of the benefit-risk profile in COVID-19 patients is urgently needed. The current review was based on very limited available data; therefore, further well-designed clinical trials are required.

Keywords: COVID-19, antiviral drugs, SARS-CoV-2, remdesivir, lopinavir/ritonavir, favipiravir

\section{Introduction}

COVID-19 is a disease resulting from a brand-new form of severe acute respiratory syndrome coronavirus 2 (SARS-CoV-2), which originated in Wuhan, China. The World Health Organization (WHO) has mentioned this disease as a state of emergency and qualifies it as a pandemic on March 11th, 2020. ${ }^{1}$ Furthermore, the global 
fatality rate (CFR) for COVID-19 is predicted to be between $2 \%$ and $3 \%$, which is almost 15 times higher than the CFR of seasonal influenza $(0.0962 \%){ }^{2}$ Also, it was discovered that by July 1st, 2020, more than 10 million people worldwide have been infected with SARS-CoV-2. ${ }^{3}$

Coronavirus $(\mathrm{CoV})$, a genus in the family Coronaviridae, is a $25-32 \mathrm{~kb}$, single-stranded enveloped ribonucleic acid (RNA) virus with a positive perception which is the largest genome among RNA viruses. ${ }^{4}$ Alpha, Beta, Gamma, and Delta are the four genera that comprise the Coronaviridae family. However, only the Alpha and Beta genera are known to be pathogens in mammals and humans, ${ }^{5}$ whereby the SARS-CoV-2 is a type of beta coronavirus. ${ }^{4}$ The viral structural spike protein (S), which binds to the angiotensin-converting enzyme 2 (ACE2) receptor, allows SARS-CoV-2 to enter and infect cells. Viral particles enter the cell via host receptors and endosomes that are attached to the receptor. ${ }^{6}$

COVID-19 is a public health emergency and currently poses a huge burden due to the dynamics of transmission and the polyphasic nature of the disease. Until now, no effective and clinically proven pharmacological treatment has been discovered. Therefore, an effective antiviral therapy strategy for SARS-CoV-2 may be beneficial in reducing the impact of COVID-19. ${ }^{7}$ Several therapeutic drugs have been investigated for COVID-19 treatment, however, no antiviral agents have been proven to be clinically effective. $^{8}$

Among several repurposed drugs currently used against SARS-CoV-2, nucleoside analogs are one of the preferred. Furthermore, the main advantages of nucleotides over nonnucleoside antiviral agents include their applicability to a broad spectrum of viral strains or species and their ability to overcome antiviral resistance. This is due to the different polymerases mechanisms, that are common to most nucleoside analogs used in antiviral therapy, and to the well-conserved nature of the nucleotide-binding sites in the polymerases among the virus families. Among synthetic drugs being studied, nucleotide precursors which include favipiravir, oxypurinol, pentoxifylline, and purine analogue derivatives, are very essential, especially in the present day. ${ }^{9}$

Nucleoside synthesis and precursors are active against Human $\mathrm{CoV}$ (HCoV) species, especially SARS-CoV-2. The mechanism is particularly relevant for spike protein (S), RNA-dependent RNA-polymerase (RdRp), papainlike proteases (PLpro), and major proteases (Mpro,
3CLpro). Therefore, administration of potential Mpro inhibitors such as lopinavir and ritonavir has often been reported for use in SARS, the Middle East respiratory syndrome (MERS), and COVID-19 patients. Also, the targeting of RdRp by antiviral drugs is a potential therapeutic option that inhibits the polymerization of coronavirus RNA and results in viral replication, ${ }^{9}$ and one of the antiviral nucleoside analogues that work on the $\mathrm{RdRp}$ inhibitor process is Remdesivir. ${ }^{10}$

Currently, many clinical studies have discussed the potential use of antiviral agents such as remdesivir, favipiravir, and lopinavir/ritonavir. ${ }^{2}$ Therefore, this article review was performed to see the effectiveness and safety of these antiviral drugs in COVID-19 treatment.

\section{Methods}

An electronic literature search was carried out on MEDLINE (PubMed) and Cochrane Library up until July 2021 to discover articles that provide information on the effectiveness of antiviral drugs such as remdesivir, lopinavir/ritonavir, and favipiravir for COVID-19. Furthermore, The Preferred Reporting Items for Systematic Review and Meta Analyses (PRISMA) checklist was used for this systematic review (Supplementary Material File 1). Also, the Mesh terms and free text words were used to search articles on the database. There were three search strings, which includes COVID-19, antiviral therapy, and effectiveness. The following keywords were used for the search, ((“COVID-19”[Mesh]), “SARS-CoV-2”[Mesh], COVID 19 Symptom*[tw] AND ((((“Antiviral Agents”[Mesh])), "remdesivir" [Supplementary Concept]), "lopinavirritonavir drug combination" [Supplementary Concept], "favipiravir" [Supplementary Concept]) AND (("adverse effects" [Subheading]), "Drug-Related Side Effects and Adverse Reactions" [Mesh]), "Treatment Outcome" [Mesh]). The detail of the search terms in all databases is provided in the Supplementary Material File 2.

All search records were collected and checked for duplicates. The selection was conducted in two stages, which include the title and abstract followed by the full-text screening processes that were performed by WFQ, DNP, and SHA, after which the difference of opinion was resolved with VFP and NZ. The following inclusion criteria was used for the screening purposes, ie, published randomized controlled trials (RCT) assessing the effectiveness of one of the antiviral drugs (remdesivir, lopinavir/ritonavir, and favipiravir) for the treatment of COVID-19 and were published in English. The exclusion criteria include the pre-clinical 
studies on animals and cells, any protocol articles, review articles, conference proceedings, and non-English studies.

\section{Risk of Bias Assessment}

The Cochrane Risk of Bias 2 (RoB 2) tool for RCTs was used to assess the risk of bias in the included studies using five domains such as randomization process, deviations from intended interventions, missing outcome data, measurement of the outcome, and selection of the reported result. ${ }^{11}$ The overall bias for each study was then classified as high, based on the criteria listed in the RoB 2 detailed guideline. $^{12}$ The graph was then derived from this tool, subsequently.

\section{Result}

\section{Study Selection and Systematic Search}

The article selection process is depicted in the PRISMA flow chart in Figure 1. The initial search retrieved 158 articles from PubMed and Cochrane Library, and after the removal of 43 duplicates, 115 articles were screened based on title and abstract and a total of 97 were removed. According to the inclusion criteria mentioned above, 18 articles were included in the full-text screening. Finally, after the removal of 3 studies, a total of 15 studies met the final inclusion criteria, thus were included in the review (Figure 1).

\section{Antiviral Therapy for COVID-19 Treatments}

A narrative summary of RCT results is presented in Table 1 to show the main characteristics of the included studies, in which 6 out of the final 15 were assessing the effectiveness of remdesivir, while three of them were focused on lopinavir/ritonavir, and seven studies were evaluating favipiravir.

\section{Remdesivir}

In this systematic review, five of six articles ${ }^{8,13-16}$ showed better clinical improvement in patients treated with remdesivir, although two of them ${ }^{13,16}$ did not provide a significant difference in comparison. Furthermore, a single article showed that combining treatment with baricitinib gave significantly better results than remdesivir alone. ${ }^{17}$ However, in terms of safety, two of six articles showed that administration of remdesivir resulted in higher mortality and more side effects than the comparison $^{14,15}$ and one article showed that the side effects in patients were not related to drug intervention. ${ }^{13}$

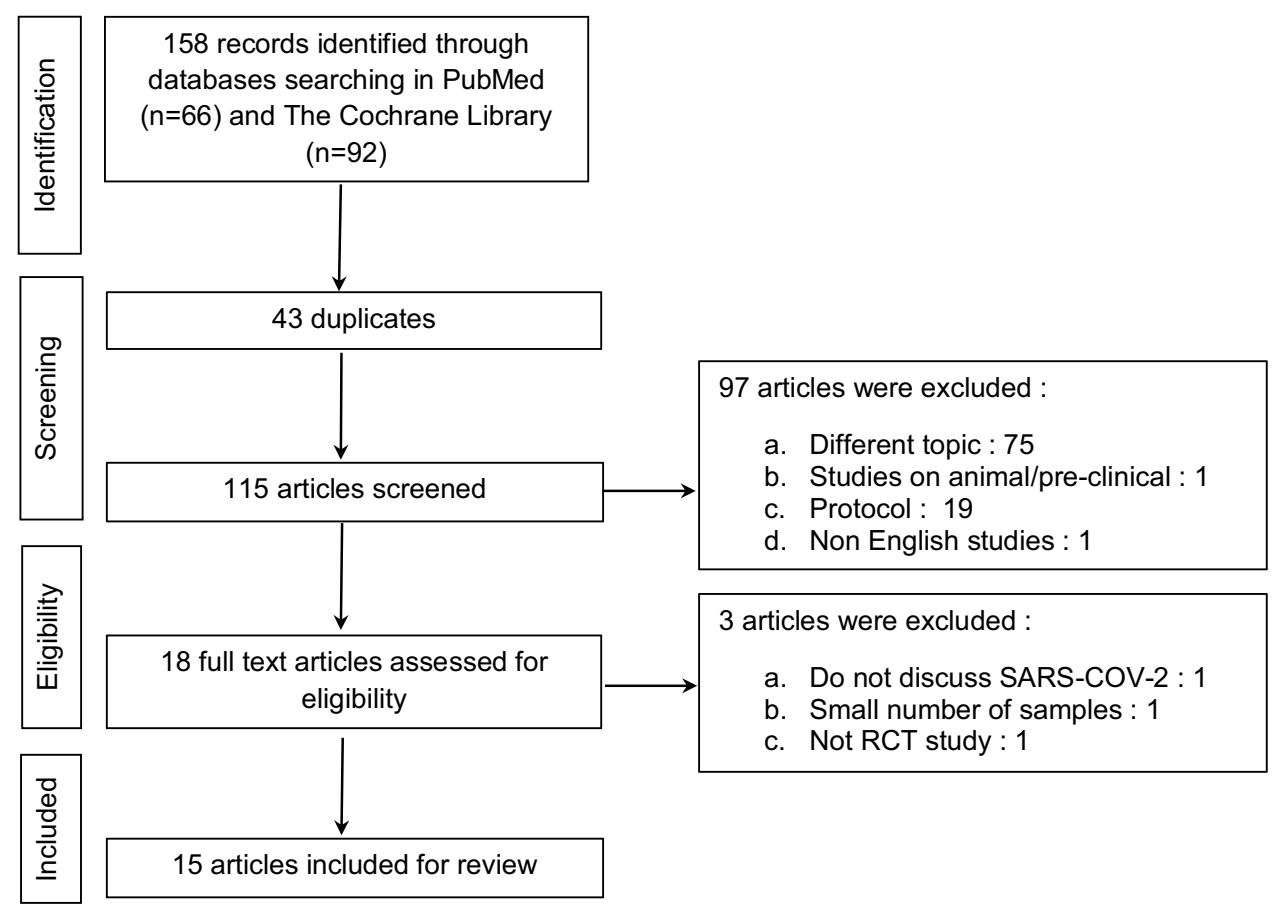

Figure I PRISMA flow diagram depicting the process of the study selection.

Note: Adapted from Page MJ, McKenzie JE, Bossuyt PM, et al. The PRISMA 2020 statement: an updated guideline for reporting systematic reviews. BMJ. 202I;372:n7I. ${ }^{60}$ Abbreviations: SARS-CoV-2, severe acute respiratory syndrome coronavirus 2; RCT, randomized controlled trial. 


\begin{tabular}{|c|c|c|c|c|c|}
\hline & 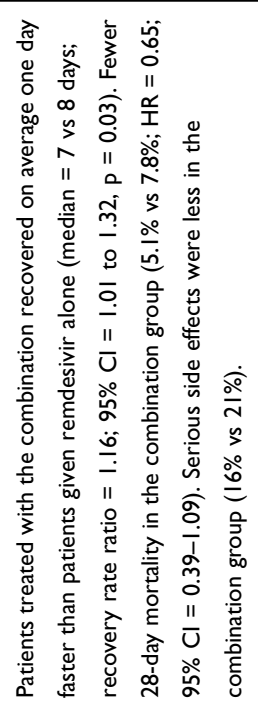 & 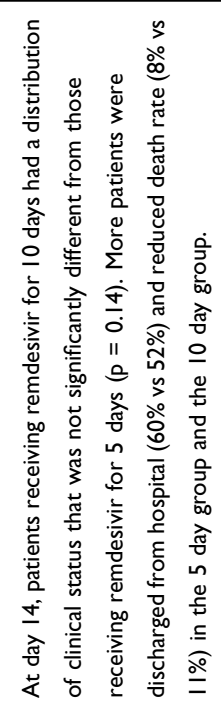 & 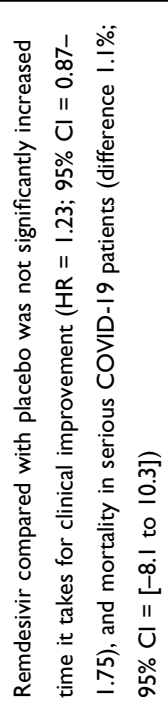 & 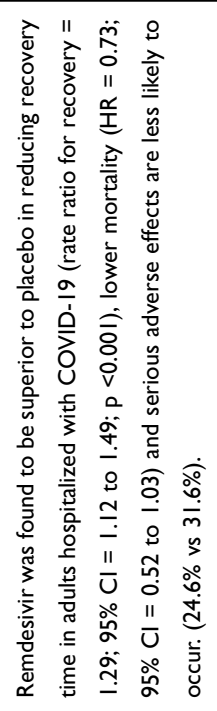 & 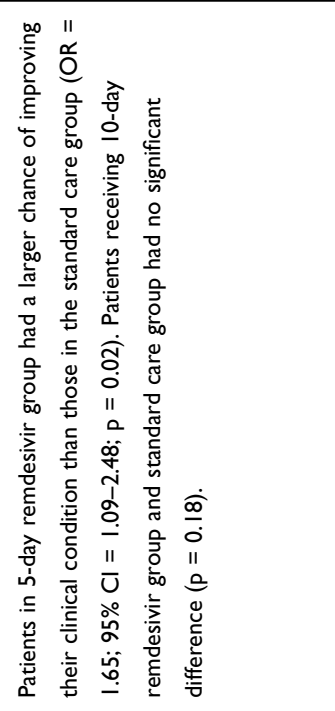 \\
\hline 总亡 & 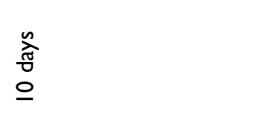 & 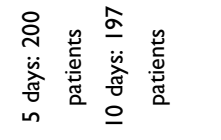 & $\begin{array}{l}\text { 促 } \\
\text { o } \\
\underline{0}\end{array}$ & 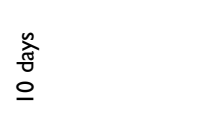 & 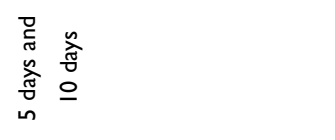 \\
\hline 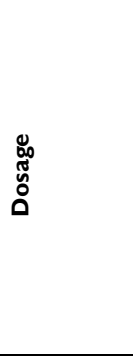 & 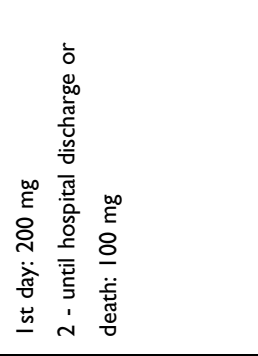 & 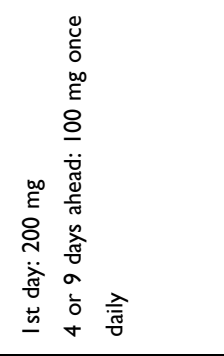 & 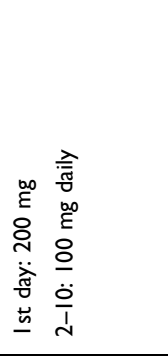 & 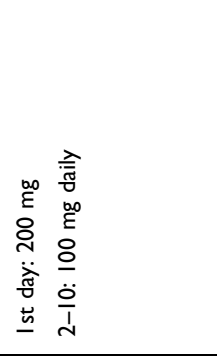 & 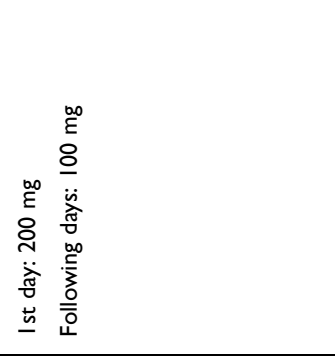 \\
\hline 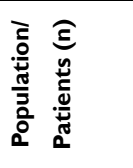 & 。్ & 放 & $\stackrel{\stackrel{\infty}{\Omega}}{ }$ & 匩 & 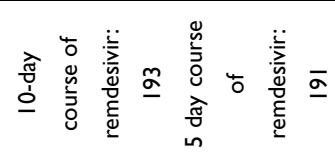 \\
\hline ù & 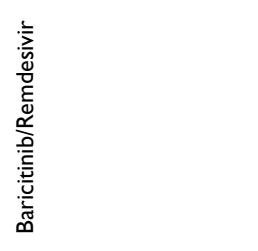 & $\widehat{\widehat{z}}$ & 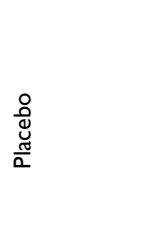 & $\begin{array}{l}\stackrel{\circ}{\mathbb{Q}} \\
\frac{\mathbb{\pi}}{0} \\
\frac{0}{0}\end{array}$ & 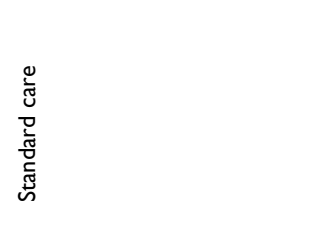 \\
\hline 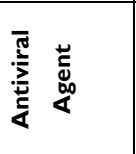 & 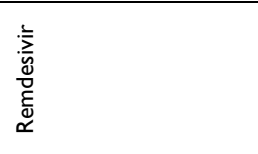 & 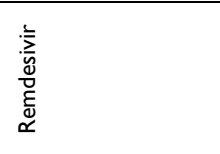 & 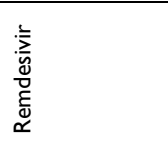 & 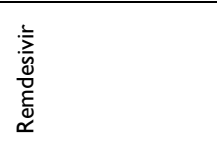 & 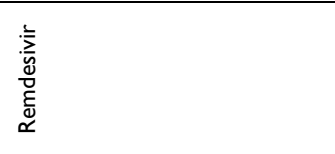 \\
\hline 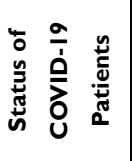 & 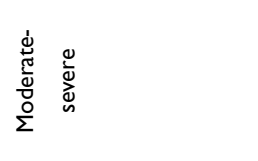 & 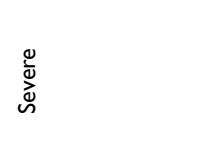 & 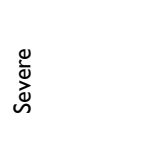 & 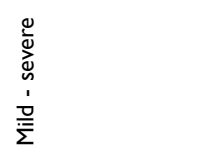 & 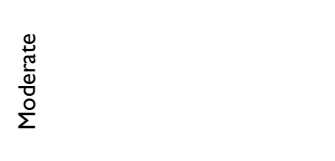 \\
\hline 造 & $\underline{\underline{m}}$ & $\tilde{\sigma}$ & $\widehat{\approx}$ & $\underline{\widetilde{o}}$ & 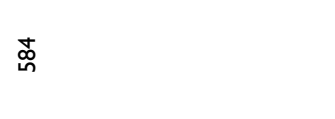 \\
\hline 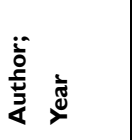 & 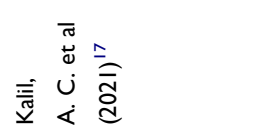 & 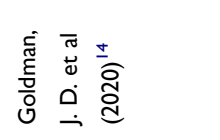 & 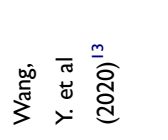 & 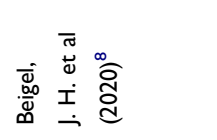 & 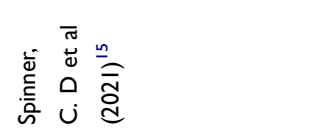 \\
\hline
\end{tabular}




\begin{tabular}{|c|c|c|c|c|c|}
\hline 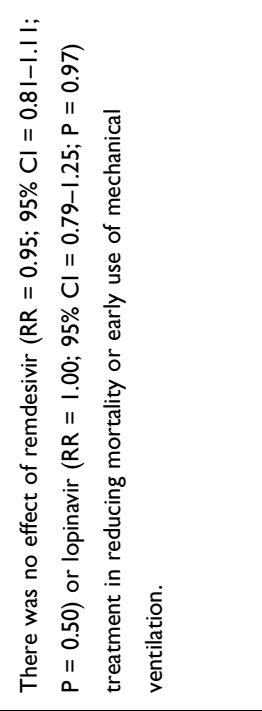 & 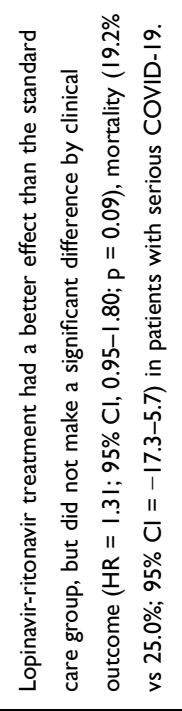 & 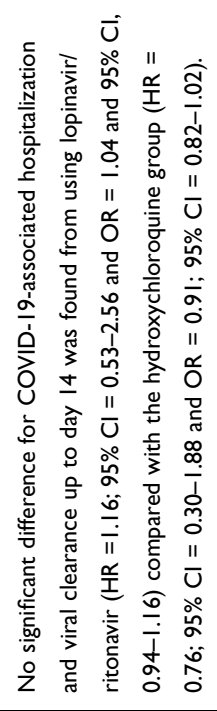 & 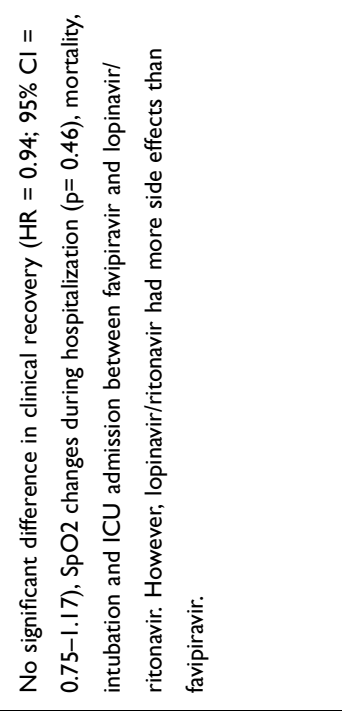 & 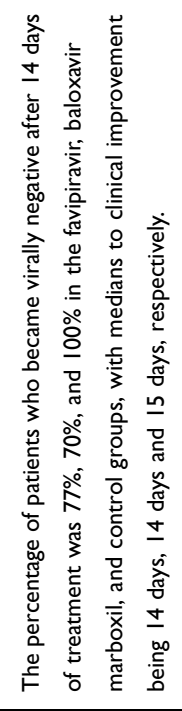 & 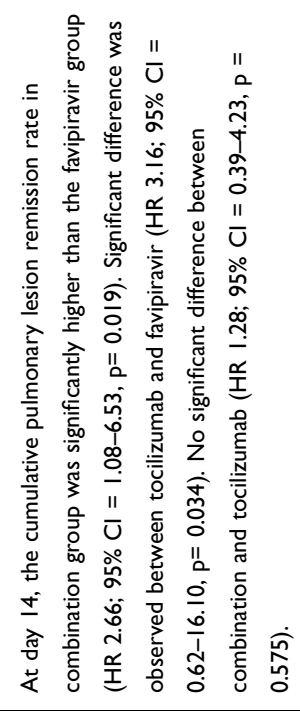 \\
\hline 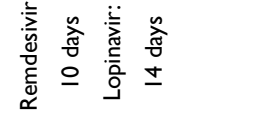 & $\begin{array}{l}\text { 蒿 } \\
\underline{0} \\
\underline{ \pm}\end{array}$ & $\begin{array}{l}\text { 令 } \\
\text { 을 }\end{array}$ & 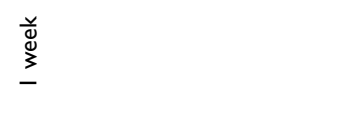 & $\begin{array}{l}\text { 令 } \\
\underline{0} \\
\underline{t}\end{array}$ & $\begin{array}{l}\text { 瓷 } \\
\underline{ \pm}\end{array}$ \\
\hline 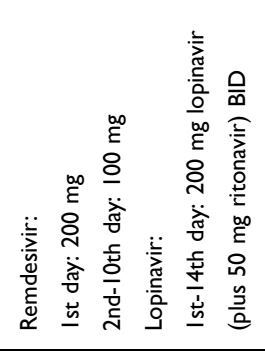 & 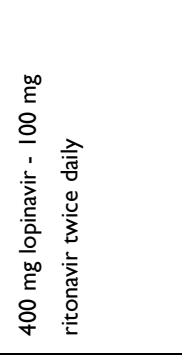 & 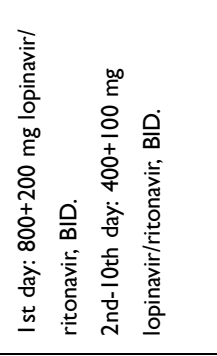 & 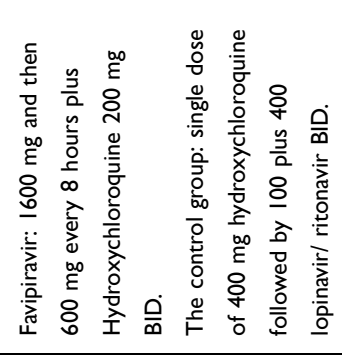 & 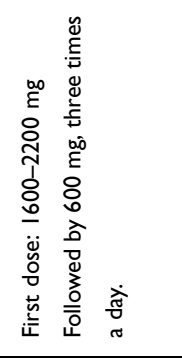 & 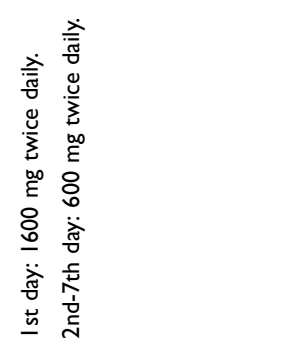 \\
\hline 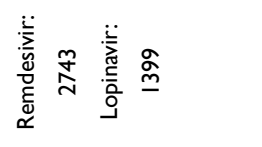 & $\sigma$ & $\underset{\sim}{ \pm}$ & 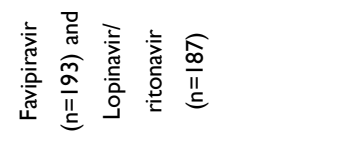 & $a$ & 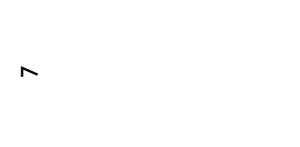 \\
\hline & 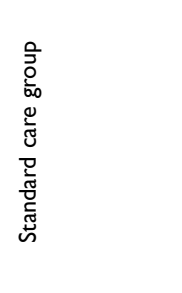 & 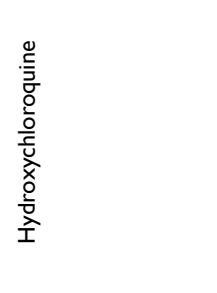 & 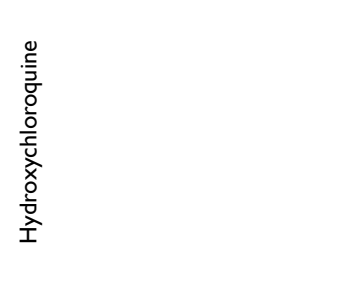 & 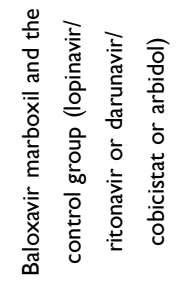 & 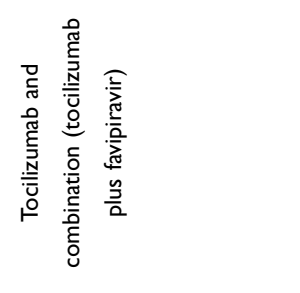 \\
\hline 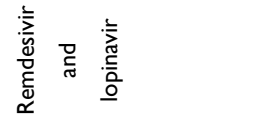 & 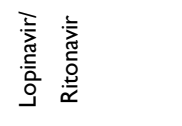 & 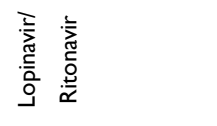 & 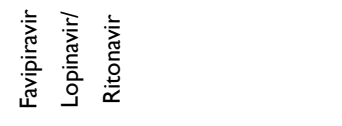 & 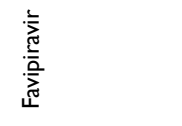 & 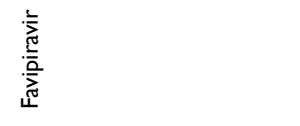 \\
\hline$\widehat{\Sigma}$ & 芯 & $\widehat{\Sigma}$ & 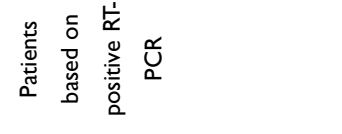 & $\frac{\widehat{s}}{\mathrm{z}}$ & $\widehat{\Sigma}$ \\
\hline $\begin{array}{l}\stackrel{\circ}{=} \\
=\end{array}$ & $\stackrel{\sigma}{\underline{\sigma}}$ & $\stackrel{\stackrel{\mu}{0}}{0}$ & $\underset{m}{\infty}$ & ০ & $\stackrel{2}{\text { N }}$ \\
\hline 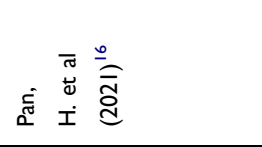 & 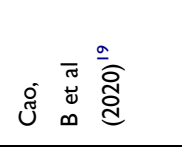 & 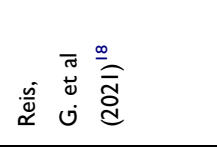 & 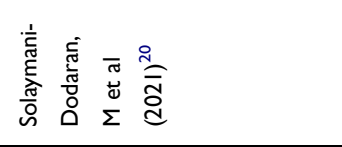 & 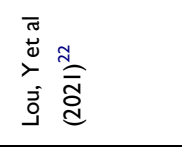 & 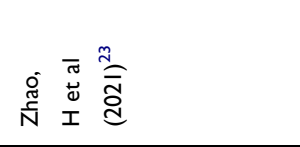 \\
\hline
\end{tabular}




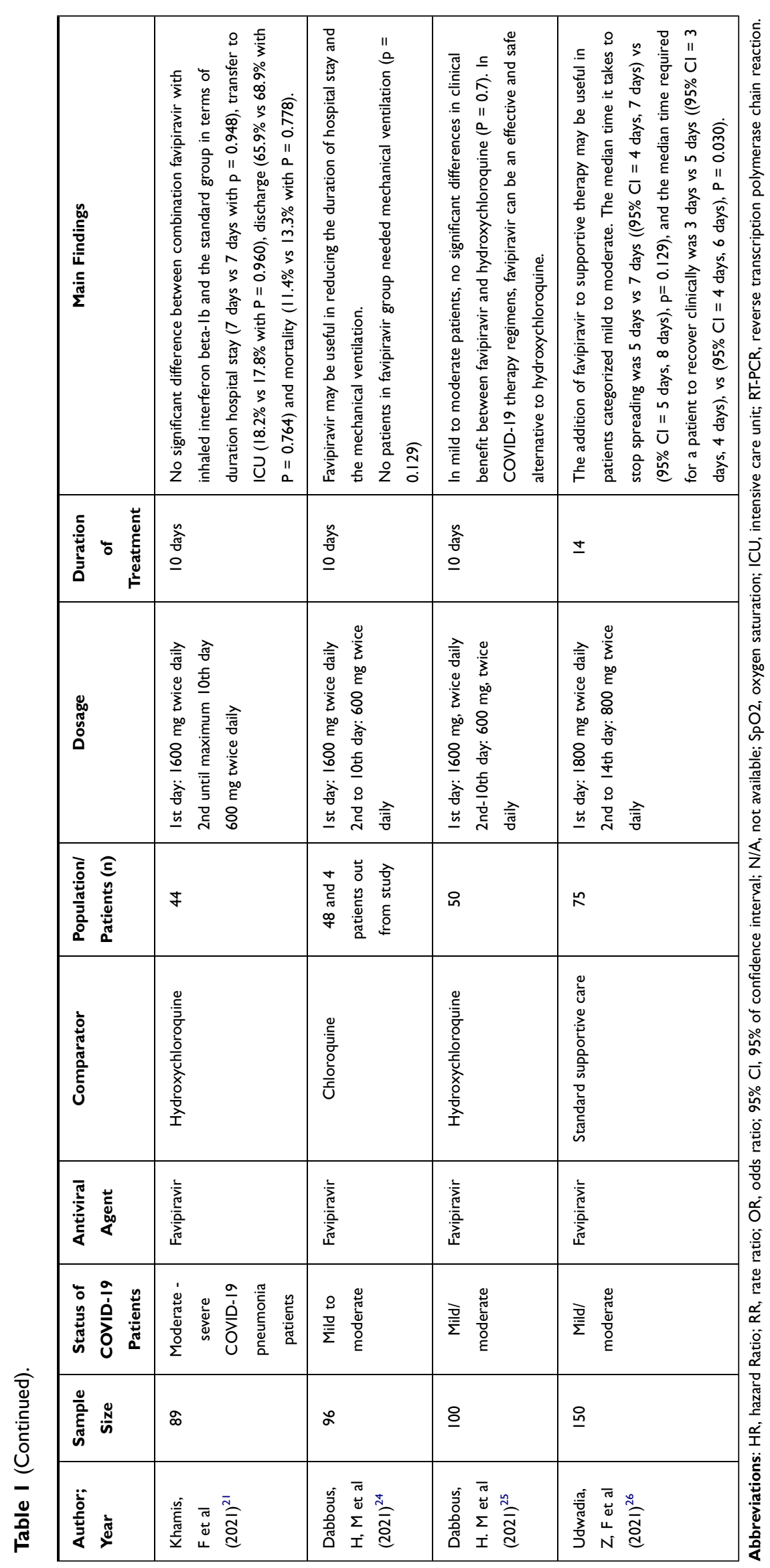


Wang et al investigated the clinical improvement of adult patients that were hospitalized and administered intravenous remdesivir for 10 days. A total of 237 COVID-19 patients were randomly recruited for this randomized, double-blind, placebo-controlled trial with 158 for the remdesivir group and 79 for the placebo group. The results showed that remdesivir did not significantly increase clinical improvement, and viral clearance in patients but showed a slightly better clinical improvement time than placebo in patients receiving remdesivir within 10 days of symptom onset in the ITT (Intention-To-Treat) population (median 18.0 days [IQR (Interquartile range) 12-28] vs 23 days [IQR 15-28]; HR [Hazard Ratio], 1.52 [95\% CI [Confidence Interval] 0.95-2.43]). Between the two groups, there was no significant difference in the clinical improvement time (median 21 days [IQR 13-28] for the remdesivir group vs 23 days [IQR 15-28] for the placebo group; HR 1.23 [95\% CI 0.87-1.75]). At 28 days, the remdesivir group had a higher mortality rate than the placebo ( $14 \%$ vs $13 \%)$, but all deaths that occurred during the follow-up were assessed to be unrelated to the intervention. Side effects were experienced by $66 \%$ and $64 \%$ of those that were administered remdesivir and placebo, respectively. Subsequently, more patients in the remdesivir group discontinued therapy because of side effects than those in the placebo $(12 \%$ vs $5 \%) .{ }^{13}$

Spinner et al and Goldman et al also investigated the effectiveness of remdesivir therapy for the period of 5 and 10 days. Both discovered no significant difference between 5 days versus 10 days remdesivir, ${ }^{14,15}$ especially during recovery and the period at which the oxygen support is stopped. ${ }^{15}$ However, in terms of clinical status, the 5-day remdesivir arm substantially outperformed the standard care arm with an odds ratio (OR) of 1.65 ; 95\% CI 1.09 2.48; $\mathrm{P}=0.02 .{ }^{14,15}$ According to ${ }^{14}$ the hospital discharge time for the 5-day treatment group was shorter $(60 \%)$. In both studies, side effects occur more frequently in the 10day treatment group in both studies, but serious side effects were more frequent in the standard care group $(9 \%$ vs $5 \%){ }^{15}$ The most common of these side effects reported include nausea, hypokalemia, and headaches. ${ }^{15}$ Furthermore, the mortality on day 28 was recorded among $1 \%$ of the patients in the 5-day care group, $2 \%$ in 10 -day, and $2 \%$ in the standard care group. ${ }^{15}$ The results of Goldman's study of the benefits cannot be determined because there was no placebo control in the study. ${ }^{14}$

Beigel et al evaluated remdesivir in 1062 adult patients with COVID-19 in a double-blind, randomized, and placebo-controlled trial with 541 were given remdesivir and 521 were administered placebo. Furthermore, patients treated with remdesivir had shorter recovery time ( 10 vs 15 days with a recovery ratio of $1.29 ; 95 \%$ CI, $1.12-1.49$; $\mathrm{P}<0.001)$, lower mortality $(6.7 \%$ vs $11.9 \%$ at day $15 ; 11.4 \%$ vs $15.2 \%$ at day 29 ; HR $0.73 ; 95 \%$ CI, $0.52-1.03$ ), shorter hospital stay (12 vs 17 days) and fewer serious side effects than placebo $(24.6 \%$ vs $31.6 \%)$. Remdesivir also prevents the development of respiratory disease, as evidenced by the low number of patients experiencing respiratory side effects and the low need for oxygen therapy. ${ }^{8}$

Remdesivir-related mortality was evaluated by Pan et al compared to the control group with 2743 patients received treatment with remdesivir and 2708 control patients received standard local care. The result showed that the remdesivirtreated group recorded 301 fatalities compared to 303 in the control group (relative risk (RR) 0.95 ; $95 \%$ CI $0.81-1.11$; $\mathrm{P}=0.50$ ). After randomization, ventilation initiation begins among 295 and 284 subjects in the remdesivir-treated arm and the control arm, respectively. Considering the results, remdesivir had no effect on reducing patient death or initiation of ventilation in patients with COVID-19. ${ }^{16}$

Furthermore, Kalil et al conducted a study comparing the response of baricitinib plus remdesivir to remdesivir plus placebo in hospitalized adult patients with COVID19. In terms of clinical improvement and recovery time, baricitinib plus remdesivir showed better outcomes ( 7 vs 8 days with a recovery rate of $1.16 ; 95 \% \mathrm{CI}, 1.01-1.32 ; \mathrm{P}=$ $0.03)$, 28-day mortality (5.1\% vs $7.8 \%$, hazard-to-death ratio $0.65 ; 95 \% \mathrm{CI}, 0.39-1.09)$ and serious adverse events ( $16 \%$ vs $21 \%$; difference, -5.0 percentage points; $95 \% \mathrm{CI}$, -9.8 to $-0.3 ; \mathrm{P}=0.03)^{17}$ than remdesivir alone.

\section{Lopinavir/Ritonavir}

Three articles discussed the effectiveness of lopinavir/ritonavir in the treatment of COVID-19, while two articles showed no significant benefit, ${ }^{18,19}$ but in terms of ICU length of stay, duration of hospital discharge, and clinical improvement at day 14, the lopinavir/ritonavir group was preferred. ${ }^{19}$ However, one article $^{16}$ showed no effect of lopinavir/ritonavir treatment on the mortality of COVID19 patients. In terms of safety, two articles discovered that the lopinavir/ritonavir group had a greater rate of adverse events. $^{18,19}$

Treatment of COVID-19 early in the diagnosis helps to prevent disease exacerbation. The study on the effect of early treatment with lopinavir/ritonavir in COVID-19 
patients was conducted by Reis et al. In this randomized clinical trial, 200 and 208 patients received lopinavir/ritonavir, and placebo as controls, respectively. The Cox model showed no statistically significant difference in the risk of hospitalization (based on ITT analysis, HR 1.16; 95\% CI $0.53-2.56$ band based on PP analysis, HR 1.82; 95\% CI 0.76-4.35), with the hospitalization of 14 patients (5.7\%) receiving lopinavir/ritonavir hospitalized for COVID-19, with a median time between randomization to an admission of 3.6 days (2.5-4.8 days), while in the placebo group 11 patients $(4.8 \%)$ were hospitalized due to COVID-19 with a median time between randomization to the hospitalization of 2.4 days ( $0.8-3.2$ days). In terms of virological clearance, there was no significant difference (OR 1.04; 95\% CI 0.94-1.16) and symptom resolution as assessed using the WURSS (Wisconsin Upper Respiratory Symptom Survey) scale, in which chest tightness symptom resolution time had an HR of 0.99 (95\% CI $0.65-1.51$ ), dry cough symptom resolution time had an HR of 0.76 (95\% CI, 0.51-1.13), and sore throat symptom resolution time had an HR of 0.88 (95\% CI, 0.64-1.22) based on ITT analysis. Ninety-two patients (39.7\%) treated with lopinavir/ritonavir experienced side effects, in which 20 (8.6\%) were serious, but none resulted in discontinuation of treatment. Meanwhile, in the placebo group, 46 patients (20.9\%) experienced side effects of treatment, of which $12(5.5 \%)$ were serious and none caused discontinuation of treatment. Also, 2 and 1 death were recorded in the lopinavir/ritonavir and placebo treatment group at the end of this trial. ${ }^{18}$

Cao et al compared the effectiveness of lopinavir/ritonavir to standard care with the main outcome of clinical improvement in patients. Furthermore, adult patients with COVID-19 admitted to the hospital were studied in a controlled, randomized, open-label trial. Supplemental oxygen, antibiotics, invasive and non-invasive ventilation, renal replacement therapy, vasopressor support, and extracorporeal membrane oxygen (ECMO) if needed were all part of the standard of treatment. According to this study, although the results obtained did not significantly provide a better clinical outcome for lopinavir/ritonavir than standard care alone, the administration of lopinavir/ritonavir showed similar clinical improvement as patients in the standard care group (median, 16 vs 16 days; hazard to clinical improvement 1.31; 95\% CI, 0.95-1.80, $\mathrm{P}=0.90$ ). The lopinavir/ritonavir arm had a reduced 28-day mortality rate than the standard group $(19.2 \%$ vs $25.0 \%$; $95 \%$ CI, 17.3-5.7). Furthermore, it also has a shorter ICU stay (median, 6 vs 11 days; $95 \% \mathrm{CI},-9$ to 0 ) and less time in the hospital (median, 12 vs 14 days; $95 \%$ CI, 0-3) than the standard care arm. On day 14, patients in the lopinavir/ ritonavir arm showed a higher rate of clinical improvement than those in the standard arm $(45.5 \%$ vs $30 \%$; $95 \% \mathrm{CI}$, 2.2-28.8). However, there were no notable variations in the terms of death time or oxygen treatment duration. Adverse events at day 28 occurred in almost half the number of the patients in both arms, with 51 , and 32 subjects in the lopinavir/ritonavir and standard group experiencing severe adverse events. Gastrointestinal side effects such as vomiting, diarrhea, and nausea were more prevalent in the lopinavir/ritonavir arm. ${ }^{19}$

Pan et al evaluated the mortality of patients receiving lopinavir/ritonavir. Furthermore, 1399 patients received lopinavir/ritonavir therapy and 1372 patients as controls received standard care. The results showed 148 death of patients in the lopinavir/ritonavir-treated group and 145 deaths in the control group (rate ratio 1.00; 95\% CI $0.79-1.25 ; \mathrm{P}=0.97$ ). Also, a total of 126 patients were ventilated in the lopinavir/ritonavir-treated group and 121 patients in the control group. Therefore, based on this study, treatment with lopinavir/ritonavir had no influence on mortality and ventilation initiation in COVID-19 patients. ${ }^{16}$

\section{Favipiravir}

Seven included articles that discussed the effectiveness of favipiravir in treating COVID-19 showed conflicting results. Three articles ${ }^{20-22}$ showed that favipiravir was not significantly different from the control group. Meanwhile, one article ${ }^{23}$ showed that the combination of favipiravir and tocilizumab produced better results. In addition, three articles ${ }^{24-26}$ showed that favipiravir was better than standard therapy (chloroquine and hydroxychloroquine), and it was also better when added as a supportive therapy.

Solaymani-Dodaran et al compared the addition of favipiravir and lopinavir/ritonavir (control) to the standard of care. The study was conducted among 373 patients, and the results showed mortality in 47 patients, of which 26 , and 21 were favipiravir and lopinavir/ritonavir patients, respectively. Furthermore, 56 individuals were transferred to ICU (27 in the favipiravir group vs 17 in the lopinavir/ ritonavir group) and 44 of them were intubated (31 in the favipiravir group vs 25 in lopinavir/ritonavir group). The Favipiravir and lopinavir/ritonavir groups did not have any significant differences in ICU admissions (31 vs 25 
patients), duration of stay in hospital (7 vs 6 days), clinical recovery (HR $0.94 ; 95 \% \mathrm{CI}=0.75-1.17$ ), and $\mathrm{SpO}_{2}$ changes during hospitalization $(\mathrm{P}=0.46)$. However, lopinavir/ritonavir had more side effects than favipiravir, specifically gastrointestinal, allergic, and respiratory side effects. Based on these results, adding favipiravir to the standard of care was not much different from the lopinavir/ ritonavir group. ${ }^{20}$

Zhao et al studied the level of IL-6 in COVID-19 patients (inflammatory biomarker) since IL-6 has an essential role in the severity of the diseases. The results showed that 3 of 7 patients experienced a decrease in the percentage of lymphocytes and the death rate or invasive mechanical ventilation was less common in the favipiravir than in the combination group. Meanwhile, favipiravir and tocilizumab showed no significant difference. Therefore, the combination of favipiravir and tocilizumab can help COVID-19 patients with lung inflammation and mortality. On day 14, the combination group had a substantially greater cumulative pulmonary lesion remission rate than the favipiravir group ( $\mathrm{P}=0.019$, HR $2.6695 \% \mathrm{CI}[1.08$ 6.53]), in the tocilizumab and favipiravir group there was a significant difference ( $\mathrm{P}=0.034$, HR $3.16,95 \%$ CI 0.62 16.10), but not between the combination and tocilizumab groups ( $\mathrm{P}=0.575$, HR $1.2895 \%$ CI $0.39-4.23$ ). Nine patients in the combination group and 2 patients in the favipiravir and tocilizumab groups reported adverse reactions. The most common side effect was an increase in transaminases, especially after treatment with tocilizumab. However, there were no severe adverse reactions, and the patient's adverse reactions subsided within a few days. ${ }^{23}$

The study of favipiravir was also carried out by Khamis et al by combining it with inhaled interferon beta- $1 \mathrm{~b}$ which was compared to standard treatment with hydroxychloroquine. The results showed no significant differences in inflammatory markers of discharge such as C-reactive protein $(\mathrm{P}=0.413)$, lactate dehydrogenase $(\mathrm{P}=$ 0.259), ferritin $(\mathrm{P}=0.968)$, and IL-6 $(\mathrm{P}=0.410)$; transfer to ICU (18.2 vs $17.8 \%, \mathrm{P}=0.960$ ), duration hospital stay ( 7 days vs 7 days, $\mathrm{P}=0.948$ ), decreased in oxygen saturation $(94 \%$ vs $95 \%)$ and mortality $(11.4 \%$ vs $13.3 \%, \mathrm{P}=$ 0.778 ) between the two groups. ${ }^{21}$

Dabbous et al and Dabbous et al conducted studies comparing favipiravir with chloroquine ${ }^{24}$ and hydroxychloroquine. ${ }^{25}$ The results of the two studies showed no significant difference between the favipiravir and control groups. In general, it was suggested that the favipiravir was better than the control, ${ }^{24,25}$ and it also had a shorter hospital stay compared to the control group $\left(13.29 \pm 5.86\right.$ days vs $15.89 \pm 4.75$ days; $\mathrm{P}=0.060^{24}$ and 11.5 vs 12.4 days. ${ }^{25}$ Furthermore, no patient required mechanical ventilation or no patient had oxygen saturation below $90 \%(\mathrm{P}=0.129)$. In this study, 1 , and 2 patients from the favipiravir and control group died $(2.3 \%$ vs $4.2 \%),(\mathrm{P}=1.00) .{ }^{24}$ Also, there was no significant difference in D-dimer levels of over 1000 ( $6 \%$ vs $14 \%$ ), in the main signs and symptoms including fever ( $36 \%$ vs $38 \%$; $\mathrm{P}=0.275)$ and dry cough $(25 \%$ vs $30 \% ; \mathrm{P}=0.574)$, at onset the mean PCR was negative for SARS-CoV-2 (8.3 days vs 8.1 days), in which more than half of patients became PCR negative on or before day 7 of diagnosis $(48 \%$ vs $55.1 \% ; \quad \mathrm{P}=0.7)$, and on viral clearance before day $7(45.5 \%$ vs $68.2 \% ; \mathrm{P}=0.379){ }^{25}$

Udwadia et al conducted a study to determine the effectiveness of adding favipiravir to supportive therapy. The results showed that the median time it takes the SARS-CoV-2 to stop spreading (5 days vs 7 days (95\% $\mathrm{CI}=4$ days, 7 days $)$ vs $(95 \% \mathrm{CI}=5$ days, 8 days $), \mathrm{P}=$ $0.129)$, the median time to recovery from initial clinical symptoms ( 3 days vs 5 days ( $95 \% \mathrm{CI}=3$ days, 4 days) vs (95\% CI $=4$ days, 6 days), $\mathrm{P}=0.030$ ), and time to discharge from hospital $(\mathrm{P}=0.108$ and in the moderate group 0.067) in the favipiravir-treated group were more favorable and tolerant than the control group. Also, favipiravir has been observed to shorten the duration of early signs and symptoms in patients with mild-to-moderate COVID-19. ${ }^{26}$

Lou et al then conducted a study comparing antiviral activity from 3 groups as the favipiravir, baloxavir marboxil, and a control group (lopinavir/ritonavir or arbidol or darunavir/cobicistat) in adults hospitalized with COVID19. The result found that there was no significant difference between the three groups in making the virus in patients negative at 14 days (77\% for favipiravir, $100 \%$ for the control group, $70 \%$ for baloxavir marboxil). Therefore, the median time it takes for clinical improvement with favipiravir, baloxavir marboxil, and the control group were 14 days, 14 days, and 15 days, respectively. ${ }^{22}$

\section{Risk of Bias}

Figure 2 depicts the RoB assessment in the included studies. According to RoB 2 tool results, 8 studies out of 15 included RCTs (54\%) had some risk of bias, 2 studies had high risk of bias (13\%) and 5 studies were deemed low in their risk of bias assessment (33\%). In details, $93 \%$ of studies had low risk through a selection of the reported 


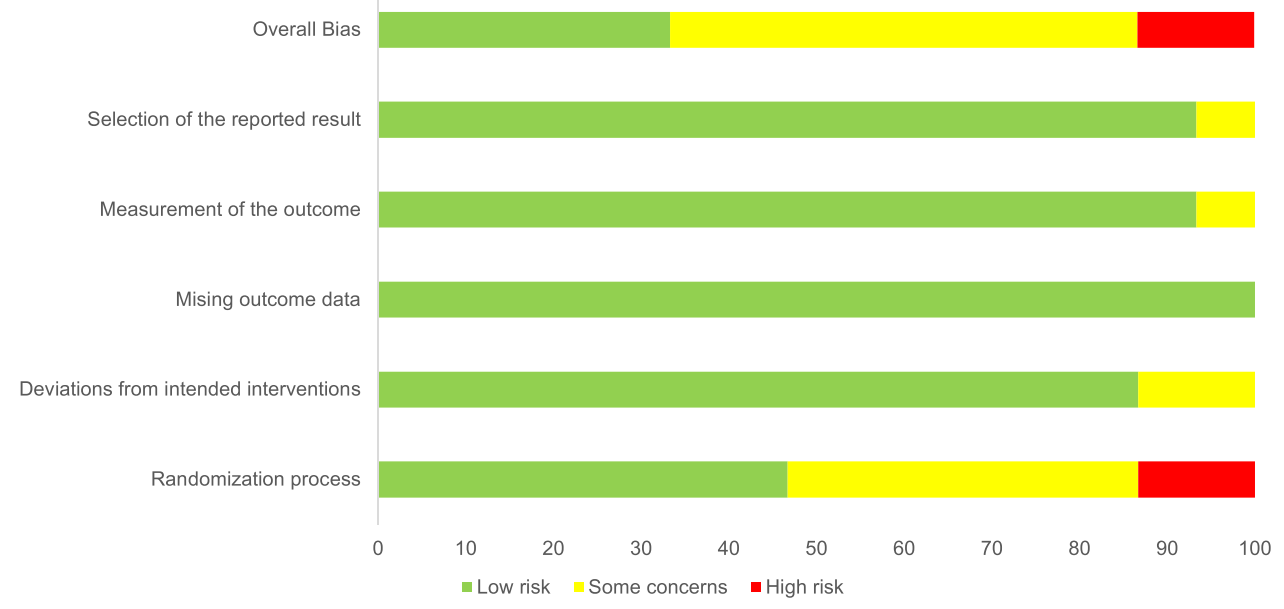

Figure 2 Risk-of-bias assessment of 15 included studies.

result, 93\% studies had low risk from measurement of the outcome, $100 \%$ had low risk of bias from missing outcome data, $87 \%$ were assessed as had a low risk of bias through deviations from intended interventions, and $47 \%$ studies had low risk of bias from randomization process.

\section{Discussion}

The new SARS-CoV-2 coronavirus, which first appeared in China at the end of 2019, has caused the deaths of more than 2 million people $(2,886,728)$ by April 7, 2021, ${ }^{27}$ with enormous losses for global health and economy. ${ }^{28}$ This systematic review was conducted to assess three antivirals as potential treatments for COVID-19, ie, remdesivir, lopinavir/ritonavir, and favipiravir. The results from this review suggested that remdesivir, in general, was potentially beneficial in improving clinical improvement of hospitalized COVID-19 patients, although some uncertainties remain on its effect on reducing mortality. Favipiravir alone or in combination with other supportive therapy has shown to be a promising option in improving clinical recovery for these patients. However, some results showed that there were no significant differences when compared with the control group. In addition, the results suggested that there was no significant clinical improvement for COVID-19 patients treated with lopinavir/ritonavir. These results mainly based on very limited clinical trials. Therefore, it is currently difficult to obtain any conclusive recommendation regarding the use of these antivirals in the clinical setting. A thorough assessment of the benefit-risk profile of these antiviral drugs for COVID-19 is urgently needed to provide a comprehensive view of the perceived clinical importance in these patients. The impact of an effective antiviral on the transmission of this virus through changing viral load and infectiousness of infected people during quarantine has to be studied in large-scale clinical trials. ${ }^{29}$ Several studies have shown promising results from existing antivirals as a therapy for SARS-CoV-2. ${ }^{3}$

Remdesivir is an RNA-dependent RNA polymerase (RdRp) inhibitor developed for the treatment of Ebola virus infection and is currently being tested for SARSCoV-2 infection treatment. ${ }^{3}$ Furthermore, it has been approved by the FDA and recommended for the treatment of hospitalized COVID-19 patients aged 12 years and above and weighs at least $40 \mathrm{~kg}$. It should only be administered in a hospital or in a health care setting capable of providing acute care comparable to inpatient hospital care. ${ }^{30}$ It was also affirmed that remdesivir is currently prescribed as one of the main treatments for COVID-19 in about 50 countries, ${ }^{31}$ and the maximum recommended daily dose is approximately $250 \mathrm{mg} / \mathrm{kg}$ of solvent used to dissolve the drug. ${ }^{32}$

The previous review stated that remdesivir is favorable in patients with severe COVID-19 compared to placebo, although the safety data on the use of remdesivir is currently sparse. ${ }^{33}$ The systematic review and network meta-analysis conducted by Lai et al also showed that remdesivir helps to improve clinical outcomes of hospitalized patients and demonstrates a promising role in treating patients with COVID-19. ${ }^{34}$ These results were in line with our review where remdesivir showed better results in terms of clinical improvement. However, it has no recommendation in patients requiring supplemental 
oxygen. In the ACTT-1 (Adaptive COVID-19 Treatment Trial) no benefit in primary outcome clinical recovery (recovery rate ratio $0.9895 \%$ CI 0.70 to 1.36 ) was observed in patients starting remdesivir while they were already on mechanical ventilation or extracorporeal membrane oxygenation. ${ }^{35}$

After the emergence of the new variant of SARS-CoV -2 variants, it is important to assess the efficacy of remdesivir against these variants, as it is the only antiviral agent approved by the FDA for the treatment of hospitalized COVID-19 patients. Furthermore, one study analyzed the protein sequence and RdRp of SARS-CoV-2 as it is a target site for several vaccines and drugs including remdesivir. Among the emerging variants, a mutation was reported in one of the remdesivir-binding residues in nsp12. This high conservation of remdesivir-binding residues did not show any evidence for remdesivir-resistant mutations. ${ }^{36}$

Another repurposed antiviral that was assessed as a potential treatment for COVID-19 was lopinavir/ritonavir. Furthermore, this drug was an anti-HIV drug that is used to treat SARS-CoV-2, and its therapeutic effect is attributed to its ability to inhibit coronavirus a 3CLpro (3C-like proteinase). ${ }^{1}$ In a non-randomized open-label experiment, lopinavir/ritonavir was shown to enhance clinical outcomes in SARS and MERS patients by inhibiting the 3CLpro (3C-like proteinase). ${ }^{37}$ Meanwhile, Lopinavir/ ritonavir was the first anti-HIV drug, which was confirmed by the National Health Commission of China in the "Guidelines for the diagnosis and treatment of COVID19 (Trial Edition 7)". 1

The results of the review showed that there was no significant difference between lopinavir/ritonavir which did not affect the mortality rate, and the control group. In the study conducted by Horby and Martin (2021), lopinavir/ ritonavir was not associated with a reduction in 28-day mortality, duration of hospital stay, or risk of progression to invasive mechanical ventilation or death. These results were consistent across subgroups of age, gender, ethnicity, duration of symptoms, number of rescue breaths, and estimated risk of death. ${ }^{38}$ It also showed no significant benefit in reducing the negative time of reverse transcription polymerase chain reaction (RT-PCR), or chest CT clearance in patients with COVID-19. ${ }^{18,39}$ In addition, more patients treated with lopinavir/ritonavir progressed from mild/moderate to severe/critical status than patients in the arbidol and placebo groups. ${ }^{40}$ Lopinavir/ritonavir improves lung function but cannot reduce viral replication. ${ }^{41}$ Also, it causes impaired renal function such as electrolyte, acid-base disturbances, and induces renal morphology. Additionally, lopinavir/ritonavir inhibits CYP enzyme binding, which in turn causes adverse drug reactions and increases the frequency and severity of other potential complications. ${ }^{42}$ Lopinavir/ritonavir also affects the body's immunoregulation which causes the initial growth of the virus to increase due to decreased immune function. ${ }^{43}$

In a study conducted by Grimaldi et al, there was a higher incidence of acute kidney injury and the need for renal replacement therapy in patients receiving lopinavir/ritonavir, which raised doubts about its safety profile. ${ }^{44}$ Likewise, lopinavir/ritonavir proved inadequate to treat the pediatric population because the number of adverse events was significantly higher in this group compared to those receiving standard therapy. ${ }^{45}$

However, several studies stated that lopinavir/ritonavir showed a favorable outcome in terms of length of stay in the ICU, hospital discharge time, and clinical improvement at day $14 .{ }^{19}$ Lopinavir/ritonavir monotherapy may also provide little benefit for improving clinical outcomes in patients hospitalized with mild/moderate COVID-19 over supportive care. ${ }^{40}$ Due to a very limited published RCT on lopinavir/ritonavir, it was impossible to draw a conclusive recommendation on the effectiveness of this drug for COVID-19 patients. Meanwhile, patients treated with lopinavir/ritonavir have multiple effects on gastrointestinal symptoms. Also, the side effects of short-term use of lopinavir/ritonavir mainly include diarrhea, abnormal stools, abdominal pain, nausea, vomiting, and asthenia. Therefore, since these side effects are capably worsening the disease, lopinavir/ritonavir treatment needs to be carefully considered in terms of risks and benefits. ${ }^{40}$

Currently, favipiravir is approved and utilized as a COVID-19 treatment in several countries. ${ }^{29}$ Based on published data and literature, countries using favipiravir are China, Hungary, India, Korea, Poland, Portugal, Russia, Serbia, Thailand, and Turkey. There is no evidence that favipiravir reduces mortality or the use of mechanical ventilation among moderate and severe COVID-19 patients. ${ }^{46}$ Previous in silico study suggested, F-RTP, which is the active form of favipiravir binds to the RdRp active site of SARS-CoV-2, SARS-CoV, and MERS-CoV in the presence of agents and proteins. ${ }^{47}$ Favipiravir is a purine nucleic acid analog licensed for the treatment of influenza since it efficiently inhibits influenza, norovirus, and Ebola viruses' RNA-dependent RNA Polymerase (RdRp). Therefore, favipiravir has the potential to 
inactivate SARS-CoV-2. ${ }^{4}$ Furthermore, it is converted intracellularly to its active phosphorylated form and then recognized as a substrate by viral $\mathrm{RdRP}^{48}$ Also, its main function is to prevent the entry and exit of viruses from cells by binding and inhibiting RdRp, which in turn prevents transcription and replication of viral genomic RNA. $^{49}$

Favipiravir has been shown to be effective against positive-stranded RNA viruses, for example, norovirus and flavivirus. Therefore, it has antiviral activity against SARS-CoV-2. ${ }^{50}$ The results showed that favipiravir has the advantage of improving the clinical status of COVID-19 patients. Also, it showed better results when used alongside supportive therapy, therefore it is used for mild to moderate patients. In addition, it was evident that Favipiravir showed a favorable outcome when combined with tocilizumab and supportive therapy. Several references have also shown that favipiravir alone might result in similar clinical improvement comparable to hydroxychloroquine. ${ }^{21,25}$ Favipiravir induces viral clearance within 7 days and contributes to clinical improvement within 14 days, and it has strong potential in the treatment of COVID-19, especially in patients with mild-tomoderate disease. An article showed that favipiravir has a tolerable safety profile in terms of total and serious side effects compared to other drugs used for short-term treatment. $^{51}$ The early viral clearance contributed to an increase in chest CT at Day $14 .^{52}$ Favipiravir also showed encouraging results with a significant decrease in the duration of viral clearance. ${ }^{53}$

Further safety concerns of favipiravir are increased blood uric acid and potential teratogenicity. ${ }^{54}$ In addition, favipiravir or its metabolites have been detected in semen and breast milk. ${ }^{55}$ Hence, the use of favipiravir in pregnant women or may become pregnant is contraindicated. Appropriate use of contraception is recommended up to 7 days after the end of treatment, for women that have childbearing potential. The use of favipiravir in pediatrics is not recommended based on the results of animal toxicity studies. $^{56}$

In some studies, antiviral drugs have been administered concurrently with or compared with hydroxychloroquine. This drug is a 4-aminoquinoline compound that has been used as an antimalaria for many years. Due to its low cost and oral administration, this drug has been considered as a potential repurposed drug candidate for treating COVID19 patients. However, to date, the use of hydroxychloroquine alone has not shown any benefit in the treatment of hospitalized COVID-19 patients. Moreover, in June 2020, the FDA revoked the permit for emergency use of hydroxychloroquine in COVID-19 patients. In summary, due to its lack of efficacy and potential risks, the use of hydroxychloroquine alone in COVID-19 patients is not recommended. ${ }^{45}$

Currently, there are no definitive treatment guidelines for COVID-19. Furthermore, the WHO has developed a living guideline that conforms to reliable standards and methods for the management of this virus. The fifth version of the WHO and COVID-19 Therapy: living guidelines mention that remdesivir and lopinavir/ritonavir are not recommended for COVID-19 treatment. Remdesivir is not recommended for hospitalized patients except under certain conditions. It can only be considered for use when the patient is free from liver dysfunction as shown by an alanine transaminase (ALT) value $>5$ times normal and does not have renal dysfunction as shown by an estimated glomerular filtration rate (eGFR) $<30 \mathrm{~mL} / \mathrm{min}$, because it is contraindicated with these conditions. ${ }^{35,57}$ In addition, the contraindications to the use of remdesivir also include use in children, as well as pregnant or lactating women. ${ }^{58}$ Until now, its use has only been intravenously, and its availability is still relatively limited. On the other hand, lopinavir/ritonavir is not recommended for the treatment of COVID-19 of any severity or duration of symptoms as it is discovered not to improve outcomes corresponding to reduced rates of treatment need, mortality, and clinical improvement. Also, it was noted that the use of lopinavir/ritonavir causes nausea and diarrhea, which in turn can increase the risk of hypovolemia, hypotension and acute kidney injury. ${ }^{57}$ Meanwhile, favipiravir is not yet included as a WHO-recommended antiviral for COVID-19 treatment. Therefore, more research is required to further assess the efficacy of these antiviral drug and its safety in the treatment of COVID-19. Favipiravir may safe and well tolerated in short-term use, however more evidence is needed to assess the effects of long-term treatment. ${ }^{54}$ Therefore, more substantial evidence is needed to confirm its efficacy and safety. ${ }^{53}$

A huge effort is being put by the scientific community to develop effective drugs for the treatment of SARSCoV-2 infection. On April 6, 2021, it was reported that there have been eight technological platforms in the study of COVID-19 vaccines which are generally based on spike proteins as well as on mRNA and DNA that control nucleosides. According to the COVID-19 vaccine tracking platform, there are already 12 vaccines approved for 
use worldwide, but only four have published the results of Phase III clinical trials. Two of these four candidates are mRNA-based vaccines, while the other two are adenovirus-based non-replicating viral vector technology. ${ }^{59}$ The availability of these vaccines is a very important breakthrough for the prevention of the COVID-19 and probably one of the effective approaches for ending the current pandemic.

The existing literature on the effectiveness of these antivirals (remdesivir, lopinavir/ritonavir, and favipiravir) have several limitations. Firstly, most of the studies used antiviral agents in combination with other drugs, therefore their results cannot be drawn solely to the antiviral that was administered. Second, there are few RCT studies on the effectiveness of remdesivir, lopinavir/ritonavir, and favipiravir for the management of COVID-19. Furthermore, the sample size used in some studies was small, therefore it limits the generalization of the findings and does not represent the population.

\section{Conclusion}

This current review provides insights into the evidencebased role of remdesivir, lopinavir/ritonavir, and favipiravir in the treatment of COVID-19. The result on the effectiveness of currently used antiviral agents suggested that the use of these drugs in clinical trials showed conflicting results. Some studies stated that remdesivir is beneficial in improving recovery and the clinical improvement of hospitalized COVID-19 patients, although its impact in reducing mortality remains uncertain. Favipiravir has shown promising results in improving the clinical status of COVID-19 patients, although several studies suggested that there were no significant differences in some clinical parameters, eg, length of hospitalizations and clinical recovery. Combination of favipiravir with other supportive therapy such as tocilizumab for the treatment of COVID-19 showed more favorable results. Moreover, prior studies stated no significant clinical improvement between lopinavir/ritonavir compared to standard care with notable adverse effect reactions. Nevertheless, this current conclusion was based on limited clinical trials data. Also, there is currently very limited safety data for these antivirals, which need to be considered in further studies. A comprehensive assessment on both the benefit and risk of these antivirals is also urgently needed to allow a more comprehensive overview for a more informed decision of using these drugs in clinical settings. Due to limited studies on this topic, further highquality evidence from well-designed clinical trials is needed.

\section{Disclosure}

The authors report no conflicts of interest in this work.

\section{References}

1. Guan W, Lan W, Zhang J, et al. COVID-19: antiviral agents, antibody development and traditional Chinese medicine. Virol Sin. 2020;35 (6):685-698. doi:10.1007/s12250-020-00297-0

2. Abuin P, Anderson A, Ferramosca A, Hernandez-Vargas EA, Gonzalez AH. Dynamical characterization of antiviral effects in COVID-19. Annu Rev Control. 2021. doi:10.1016/j. arcontrol.2021.05.001

3. Gavriatopoulou M, Ntanasis-Stathopoulos I, Korompoki E, et al. Emerging treatment strategies for COVID-19 infection. Clin Exp Med. 2021;21(2):167-179. doi:10.1007/s10238-020-00671-y

4. Liu X, Liu C, Liu G, Luo W, Xia N. COVID-19: progress in diagnostics, therapy and vaccination. Theranostics. 2020;10 (17):7821-7835. doi:10.7150/thno.47987

5. Paules CI, Marston HD, Fauci AS. Coronavirus infections-more than just the common cold. J Am Med Assoc. 2020;323(8):707-708. doi:10.1001/jama.2020.0757

6. Sanders JM, Monogue ML, Jodlowski TZ, Cutrell JB. Pharmacologic treatments for Coronavirus disease 2019 (COVID-19): a review. $J$ Am Med Assoc. 2020;323(18):1824-1836.

7. Torneri A, Libin P, Vanderlocht J, Vandamme AM, Neyts J, Hens N. A prospect on the use of antiviral drugs to control local outbreaks of COVID-19. BMC Med. 2020;18(1):1-9.

8. Beigel JH, Tomashek KM, Dodd LE, et al. Remdesivir for the treatment of Covid-19 - final report. N Engl J Med. 2020;383 (19):1813-1826. doi:10.1056/NEJMoa2007764

9. Borbone N, Piccialli G, Roviello GN, Oliviero G. Nucleoside analogs and nucleoside precursors as drugs in the fight against SARS-CoV-2 and other coronaviruses. Molecules. 2021;26(4):1-17. doi:10.3390/ molecules 26040986

10. Munir MA, Kuganda H, Basry A. The efficacy and safety of antivirus drugs for COVID-19: a systematic review. Syst Rev Pharm. 2020;11 (7):162-166.

11. Sterne JAC, Savović J, Page MJ, et al. RoB 2: a revised tool for assessing risk of bias in randomised trials. BMJ. 2019;366:1-8.

12. Higgins JPT, Savović J, Matthew J; Page JAS on behalf of the RDG. Risk of bias tools - Current version of RoB 2 [Internet]; 2019. Available from: https://www.riskofbias.info/welcome/rob-2-0-tool/ current-version-of-rob-2. Accessed November 9, 2021.

13. Wang Y, Zhang D, Du G, et al. Remdesivir in adults with severe COVID-19: a randomised, double-blind, placebo-controlled, multicentre trial. Lancet. 2020;395(10236):1569-1578. doi:10.1016/ S0140-6736(20)31022-9

14. Goldman JD, Lye DCB, Hui DS, et al. Remdesivir for 5 or 10 days in patients with severe Covid-19. $N$ Engl J Med. 2020;383 (19):1827-1837. doi:10.1056/NEJMoa2015301

15. Spinner CD, Gottlieb RL, Criner GJ, et al. Effect of remdesivir vs standard care on clinical status at 11 days in patients with moderate COVID-19: a randomized clinical trial. J Am Med Assoc. 2020;324 (11):1048-1057. doi:10.1001/jama.2020.16349

16. Pan H, Peto R, Henao-Restrepo AM, et al. Repurposed antiviral drugs for Covid-19 - interim WHO solidarity trial results. $N$ Engl J Med. 2021;384(6):497-511. 
17. Kalil AC, Patterson TF, Mehta AK, et al. Baricitinib plus remdesivir for hospitalized adults with Covid-19. N Engl J Med. 2021;384 (9):795-807. doi:10.1056/NEJMoa2031994

18. Reis G, Moreira Silva EA, Medeiros Silva DC, et al. Effect of early treatment with hydroxychloroquine or lopinavir and ritonavir on risk of hospitalization among patients with COVID-19: the TOGETHER randomized clinical trial. JAMA Netw Open. 2021;4(4):1-14. doi:10.1001/jamanetworkopen.2021.6468

19. Cao B, Wang Y, Wen D, et al. A trial of lopinavir-ritonavir in adults hospitalized with severe Covid-19. $N$ Engl J Med. 2020;382 (19):1787-1799. doi:10.1056/NEJMoa2001282

20. Solaymani-dodaran M, Ghanei M, Bagheri M, et al. Safety and efficacy of Favipiravir in moderate to severe SARS-CoV-2 pneumonia. Int Immunopharmacol. 2021;95:107522. doi:10.1016/j. intimp.2021.107522

21. Khamis F, Al Naabi H, Al Lawati A, et al. Randomized controlled open label trial on the use of favipiravir combined with inhaled interferon beta-1b in hospitalized patients with moderate to severe COVID-19 pneumonia. Int $J$ Infect Dis. 2021;102:538-543. doi:10.1016/j.ijid.2020.11.008

22. Lou Y, Liu L, Yao H, et al. Clinical outcomes and plasma concentrations of baloxavir marboxil and favipiravir in COVID-19 patients: an exploratory randomized, controlled trial. Eur $J$ Pharm Sci. 2021;157:105631.

23. Zhao H, Zhu Q, Zhang C, et al. Tocilizumab combined with favipiravir in the treatment of COVID-19: a multicenter trial in a small sample size. Biomed Pharmacother. 2021;133:110825. doi:10.1016/j. biopha.2020.110825

24. Dabbous HM, Abd-Elsalam S, El-Sayed MH, et al. Efficacy of favipiravir in COVID-19 treatment: a multi-center randomized study. Arch Virol. 2021;166(3):949-954. doi:10.1007/s00705-02104956-9

25. Dabbous HM, El-Sayed MH, El Assal G, et al. Safety and efficacy of favipiravir versus hydroxychloroquine in management of COVID-19: a randomised controlled trial. Sci Rep. 2021;11(1):1-7. doi:10.1038/ s41598-021-85227-0

26. Udwadia ZF, Singh P, Barkate H, et al. Efficacy and safety of favipiravir, an oral RNA-dependent RNA polymerase inhibitor, in mild-to-moderate COVID-19: a randomized, comparative, open-label, multicenter, Phase 3 clinical trial. Int $J$ Infect Dis. 2021;103:62-71. doi:10.1016/j.ijid.2020.11.142

27. Mercatelli D, Holding AN, Giorgi FM. Web tools to fight pandemics: the COVID-19 experience. Brief Bioinform. 2021;22(2):690-700. doi:10.1093/bib/bbaa261

28. Arthi V, Parman J. Disease, downturns, and wellbeing: economic history and the long-run impacts of COVID-19. Explor Econ Hist. 2021;79:101381. doi:10.1016/j.eeh.2020.101381

29. Joshi S, Parkar J, Ansari A, et al. Role of favipiravir in the treatment of COVID-19. Int J Infect Dis. 2021;102:501-508. doi:10.1016/j. ijid.2020.10.069

30. Piscoya A, Ng-Sueng LF, Del Riego AP, et al. Efficacy and harms of remdesivir for the treatment of COVID-19: a systematic review and meta-analysis. PLoS One. 2020;15(12):1-19. doi:10.1371/journal. pone. 0243705

31. Gholamhoseini MT, Yazdi-Feyzabadi V, Goudarzi R, Mehrolhassani MH. Safety and efficacy of remdesivir for the treatment of COVID-19: a systematic review and meta-analysis. J Pharm Pharm Sci. 2021;24(13):237-245. doi:10.18433/ jpps31870

32. Singh S, Khera D, Chugh A, Khera PS, Chugh VK. Efficacy and safety of remdesivir in COVID-19 caused by SARS-CoV-2: a systematic review and meta-analysis. BMJ Open. 2021;11(6):1-9. doi:10.1136/bmjopen-2020-048416

33. Davies M, Osborne V, Lane S, et al. Remdesivir in treatment of COVID-19: a systematic benefit-risk assessment. Drug Saf. 2020;43(7):645-656. doi:10.1007/s40264-020-00952-1
34. Lai CC, Chen CH, Wang CY, Chen KH, Wang YH, Hsueh PR. Clinical efficacy and safety of remdesivir in patients with COVID-19: a systematic review and network meta-analysis of randomized controlled trials. J Antimicrob Chemother. 2021;76 (8):1962-1968. doi:10.1093/jac/dkab093

35. Chalmers JD, Crichton ML, Goeminne PC, et al. Management of hospitalised adults with coronavirus disease 2019 (COVID-19): a European Respiratory Society living guideline. Eur Respir J. 2021;57(4):2100048. doi:10.1183/13993003.00048-2021

36. Taha HR, Keewan N, Slati F, Al-Sawalha NA. Remdesivir: a closer look at its effect in COVID-19 pandemic. Pharmacology. 2021;106 (9-10):462-468. doi:10.1159/000518440

37. Zumla A, Chan JFW, Azhar EI, Hui DSC, Yuen KY. Coronavirusesdrug discovery and therapeutic options. Nat Rev Drug Discov. 2016;15(5):327-347.

38. Horby PW, Mafham M, Bell JL, et al. Lopinavir-ritonavir in patients admitted to hospital with COVID-19 (RECOVERY): a randomised, controlled, open-label, platform trial. Lancet. 2020;396 (10259):1345-1352.

39. Joseph BA, Dibas M, Evanson KW, et al. Efficacy and safety of lopinavir/ritonavir in the treatment of COVID-19: a systematic review. Expert Rev Anti Infect Ther. 2021;19(6):679-687. doi:10.1080/14787210.2021.1848545

40. Li Y, Xie Z, Lin W, et al. Efficacy and safety of lopinavir/ritonavir or arbidol in adult patients with mild/moderate COVID-19: an exploratory randomized controlled trial. Med. 2020;1(1):105-113. doi:10.1016/j.medj.2020.04.001

41. Siddiqi HK, Mehra MR. COVID-19 illness in native and immunosuppressed states: a clinical-therapeutic staging proposal. J Hear Lung Transplant. 2020;39(5):405-407. doi:10.1016/j.healun.2020.03.012

42. Lepage M-A, Rozza N, Kremer R, Grunbaum A. Safety and efficacy concerns of lopinavir/ritonavir in COVID-19 affected patients: a retrospective series. Clin Toxicol. 2021:1-4. doi:10.1080/ 15563650.2020 .1842882

43. Lu J-M, Zhou A-F, Zhang XB, et al. Safety and efficacy of oral lopinavir/ritonavir in pediatric patients with coronavirus disease: a nationwide comparative analysis. Eur Rev Med Pharmacol Sci. 2021;25:549-555.

44. Grimaldi D, Aissaoui N, Blonz G, et al. Characteristics and outcomes of acute respiratory distress syndrome related to COVID-19 in Belgian and French intensive care units according to antiviral strategies: the COVADIS multicentre observational study. Ann Intensive Care. 2020;10(131):1-11. doi:10.1186/s13613-020-00751-y

45. Martínez VG, Salas AA, Ballestín SS. Antiviral therapeutic approaches for SARS-CoV-2 infection: a systematic review. Pharmaceuticals. 2021;14(736):1-26.

46. Özlüşen B, Kozan Ş, Akcan RE, et al. Effectiveness of favipiravir in COVID-19: a live systematic review. Eur J Clin Microbiol Infect Dis. 2021;1-9. doi:10.1007/s10096-021-04307-1

47. Sada M, Saraya T, Ishii H, et al. Detailed molecular interactions of favipiravir with SARS-CoV-2, SARS-CoV, MERS-CoV, and influenza virus polymerases in silico. Microorganisms. 2020;8(10):1-9. doi:10.3390/microorganisms 8101610

48. Seneviratne SL, Abeysuriya V, de Mel S, de Zoysa I, Niloofa R. Favipiravir in COVID-19. Int J Progr Sci Technol. 2020;19 (2):143-145.

49. Łagocka R, Dziedziejko V, Kłos P, Pawlik A. Favipiravir in therapy of viral infections. J Clin Med. 2021;10(273):1-16. doi:10.3390/ jcm 10020273

50. Ghasemnejad-Berenji M, Pashapour S. Favipiravir and COVID-19: a simplified summary. Drug Res. 2021;71(3):166-170. doi:10.1055/ a-1296-7935

51. Manabe T, Kambayashi D, Akatsu H, Kudo K. Favipiravir for the treatment of patients with COVID-19: a systematic review and meta-analysis. BMC Infect Dis. 2021;21(1):1-13. doi:10.1186/ s12879-021-06164-X 
52. Cai Q, Yang M, Liu D, et al. Experimental treatment with favipiravir for COVID-19: an open-label control study. Engineering. 2020;6 (10):1192-1198. doi:10.1016/j.eng.2020.03.007

53. Tejaswi P, Devashish K, Prasad RR. Pharmacological effects of favipiravir on coronavirus: an update. Biomed Pharmacol J. 2021;14(2):1087-1095. doi:10.13005/bpj/2212

54. Pilkington V, Pepperrell T, Hill A. A review of the safety of favipiravir - a potential treatment in the COVID-19 pandemic? J Virus Erad. 2020;6(2):45-51. doi:10.1016/S2055-6640(20)30016-9

55. Ozudogru O, Yerlikaya E, Alayunt NO, Cambay Z. Evaluation of favipiravir treatment before intensive care in COVID-19 patients. J Teknol Lab. 2021;1:22-30.

56. Instiaty DIGAAPS, Marzuki JE, Angelia F, et al. Antiviral treatment of COVID-19: a clinical pharmacology narrative review. Med J Indones. 2020;29(3):332-345. doi:10.13181/mji.rev.204652
57. World Health Organization. Therapeutics and COVID-19: LIVING GUIDELINE. World Health Organization; 2021:1-71

58. Parasher A. COVID-19: current understanding of its pathophysiology, clinical presentation and treatment. Postgrad Med J. 2021;97 (1147):312-320. doi:10.1136/postgradmedj-2020-138577

59. Costanzo M, De Giglio MAR, Roviello GN. Anti-Coronavirus vaccines: past investigations on SARS-CoV-1 and MERS-CoV, the approved vaccines from BioNTech/Pfizer, Moderna, Oxford/ AstraZeneca and others under development against SARS-CoV-2 infection. Curr Med Chem. 2021;28:1-15. doi:10.2174/ 0929867328666210521164809

60. Page MJ, McKenzie JE, Bossuyt PM, et al. The PRISMA 2020 statement: an updated guideline for reporting systematic reviews.BMJ. 2021; 372 : n71 doi:10.1136/bmj.n71.

\section{Publish your work in this journal}

The International Journal of General Medicine is an international, peer-reviewed open-access journal that focuses on general and internal medicine, pathogenesis, epidemiology, diagnosis, monitoring and treatment protocols. The journal is characterized by the rapid reporting of reviews, original research and clinical studies across all disease areas. The manuscript management system is completely online and includes a very quick and fair peer-review system, which is all easy to use. Visit http://www.dovepress.com/ testimonials.php to read real quotes from published authors.

Submit your manuscript here: https://www.dovepress.com/international-journal-of-general-medicine-journal 